\title{
Status of the silicon pore optics technology
}

Collon, Maximilien J.; Vacanti, Giuseppe; Barriere, Nicolas M.; Landgraf, Boris; Günther, Ramses; Vervest, Mark; Voruz, Luc; Verhoeckx, Sjoerd; Babi, Ljubiša ; Keek, Laurens

Total number of authors:

41

Published in:

Optics for EUV, X-Ray, and Gamma-Ray Astronomy IX

Link to article, DOI:

$10.1117 / 12.2530696$

Publication date:

2019

Document Version

Publisher's PDF, also known as Version of record

Link back to DTU Orbit

Citation (APA):

Collon, M. J., Vacanti, G., Barriere, N. M., Landgraf, B., Günther, R., Vervest, M., Voruz, L., Verhoeckx, S., Babi, L., Keek, L., Girou, D. A., Okma, B., Hauser, E., Beijersbergen, M. W., Bavdaz, M., Wille, E., Fransen, S., Shortt, B., Ferreira, I., ... Ball, K. (2019). Status of the silicon pore optics technology. In S. L. OD., \& G. P. (Eds.), Optics for EUV, X-Ray, and Gamma-Ray Astronomy IX (Vol. 11119, pp. 111190L-1-11190L-8). SPIE - International Society for Optical Engineering. Proceedings of SPIE - The International Society for Optical Engineering https://doi.org/10.1117/12.2530696

\section{General rights}

Copyright and moral rights for the publications made accessible in the public portal are retained by the authors and/or other copyright owners and it is a condition of accessing publications that users recognise and abide by the legal requirements associated with these rights.

- Users may download and print one copy of any publication from the public portal for the purpose of private study or research.

- You may not further distribute the material or use it for any profit-making activity or commercial gain

- You may freely distribute the URL identifying the publication in the public portal 


\section{Status of the silicon pore optics technology}

\section{Maximilien J. Collon, Giuseppe Vacanti, Nicolas M. Barrière, Boris Landgraf, Ramses Günther, et al.}

Maximilien J. Collon, Giuseppe Vacanti, Nicolas M. Barrière, Boris Landgraf, Ramses Günther, Mark Vervest, Luc Voruz, Sjoerd Verhoeckx, Ljubiša Babić, Laurens Keek, David Girou, Ben Okma, Enrico Hauser, Marco W.

Beijersbergen, Marcos Bavdaz, Eric Wille, Sebastiaan Fransen, Brian Shortt, Ivo Ferreira, Jeroen Haneveld, Arenda Koelewijn, Ronald Start, Maurice Wijnperle, Jan-Joost Lankwarden, Coen van Baren, Paul Hieltjes, Jan Willem den Herder, Peter Müller, Evelyn Handick, Michael Krumrey, Miranda Bradshaw, Vadim Burwitz, Giovanni Pareschi, Sonny Massahi, Sara Svendsen, Desirée Della Monica Ferreira, Finn E. Christensen, Giuseppe Valsecchi, Paul Oliver, Ian Chequer, Kevin Ball, "Status of the silicon pore optics technology," Proc. SPIE 11119, Optics for EUV, X-Ray, and GammaRay Astronomy IX, 111190L (12 September 2019); doi: 10.1117/12.2530696

Event: SPIE Optical Engineering + Applications, 2019, San Diego, California, United States 


\title{
Status of the Silicon Pore Optics technology
}

\author{
Maximilien J. Collon*1 ${ }^{1}$, Giuseppe Vacanti ${ }^{1}$, Nicolas M. Barrière ${ }^{1}$, Boris Landgraf ${ }^{1}$, \\ Ramses Günther ${ }^{1}$, Mark Vervest ${ }^{1}$, Luc Voruz ${ }^{1}$, Sjoerd Verhoeckx ${ }^{1}$, Ljubiša Babić ${ }^{1}$, Laurens Keek ${ }^{1}$, \\ David Girou $^{1}$, Ben Okma ${ }^{1}$, Enrico Hauser ${ }^{1}$, Marco W. Beijersbergen ${ }^{1}$, Marcos Bavdaz ${ }^{2}$, Eric Wille ${ }^{2}$, \\ Sebastiaan Fransen ${ }^{2}$, Brian Shortt ${ }^{2}$, Ivo Ferreira ${ }^{2}$, Jeroen Haneveld ${ }^{3}$, Arenda Koelewijn ${ }^{3}$, \\ Ronald Start $^{3}$, Maurice Wijnperle ${ }^{3}$, Jan-Joost Lankwarden ${ }^{3}$, Coen van Baren ${ }^{4}$, Paul Hieltjes ${ }^{4}$, \\ Jan Willem den Herder ${ }^{4,11}$, Peter Müller ${ }^{5}$, Evelyn Handick ${ }^{5}$, Michael Krumrey ${ }^{5}$, Miranda Bradshaw ${ }^{6}$, \\ Vadim Burwitz ${ }^{6}$, Giovanni Pareschi ${ }^{7}$, Sonny Massahi ${ }^{8}$, Sara Svendsen ${ }^{8}$, Desirée Della Monica \\ Ferreira $^{8}$, Finn E. Christensen ${ }^{8}$, Giuseppe Valsecchi ${ }^{9}$, Paul Oliver ${ }^{10}$, Ian Chequer $^{10}$, Kevin Ball ${ }^{10}$ \\ ${ }^{1}$ cosine, Oosteinde 36, 2361 HE Warmond, The Netherlands \\ ${ }^{2}$ European Space Agency, ESTEC, Keplerlaan 1, 2200 AG Noordwijk, The Netherlands \\ ${ }^{3}$ Micronit Microfluidics B.V., Colosseum 15, 7521 PV Enschede, The Netherlands \\ ${ }^{4}$ SRON, Sorbonnelaan 2, 3584 CA Utrecht, The Netherlands \\ ${ }^{5}$ Physikalisch-Technische Bundesanstalt (PTB), Abbestr. 2-12, 10587 Berlin, Germany \\ ${ }^{6}$ MPI f. extraterrestrische Physik, Giessenbachstrasse 1, 85748 Garching, Germany \\ ${ }^{7}$ INAF Osservatorio Astronomico di Brera, Via E. Bianchi 46 I- 23807, Merate (Lc), Italy \\ ${ }^{8}$ DTU Space, Technical University of Denmark, Building 327, DK - 2800 Kgs. Lyngby, Denmark \\ ${ }^{9}$ Media Lario S.r.l., Località Pascolo, 23842 Bosisio Parini(LC), Italy \\ ${ }^{10}$ Teledyne imaging Ltd., 106 Waterhouse Lane, Chelmsford, Essex CM1 2QU, England \\ ${ }^{11}$ Anton Pannekoek Institute, University of Amsterdam, 1090 GE Amsterdam, the Netherlands
}

\begin{abstract}
Silicon Pore Optics (SPO) uses commercially available monocrystalline double-sided super-polished silicon wafers as a basis to produce mirrors that form lightweight and stiff high-resolution x-ray optics. The technology has been invented by cosine and the European Space Agency (ESA) and developed together with scientific and industrial partners to mass production levels. SPO is an enabling element for large space-based x-ray telescopes such as Athena and ARCUS, operating in the 0.2 to $12 \mathrm{keV}$ band, with angular resolution requirements up to 5 arc seconds. SPO has also shown to be a versatile technology that can be further developed for gamma-ray optics, medical applications and for material research. This paper will summarise the status of the technology and of the mass production capabilities, show latest performance results and discuss the next steps in the development.
\end{abstract}

Keywords: x-ray optics, $\mathrm{x}$-ray astronomy, silicon, wafer, stack, pore optics, $\mathrm{x}$-ray telescopes, ATHENA, ARCUS, SPO

\section{INTRODUCTION}

Silicon Pore Optics were invented more than 15 years ago [1,2]. From the very beginning the eventual mass production of the more than 100.000 mirrors required to build large x-ray telescopes was taken into account. We therefore followed, after the initial proof-of-principle trials, a holistic development path that aimed at demonstrating the entire mirror module production chain, from wafer to completed module, and that iteratively improved and still improves different aspects of that chain to steadily increase the performance and the production rate. This approach leads to a smooth transition from R\&D to mass production of the flight modules and avoids the many examples of technologies that worked well in the lab, but have big issues being scaled-up to mass manufacturing. With the selection of Athena as the second L-class mission in ESA's Cosmic Vision 2015-2025 program [3-5] the development and mass production preparation efforts [6] have been intensified and this paper summarises the status of Silicon Pore Optics and the next steps.

*m.collon@cosine.nl

Optics for EUV, X-Ray, and Gamma-Ray Astronomy IX, edited by Stephen L. O'Dell, Giovanni Pareschi, Proc. of SPIE Vol. 11119, 111190L · C 2019 SPIE · CCC code: 0277-786X/19/\$21 · doi: 10.1117/12.2530696 


\section{STATUS}

SPO is being developed by a consortium of industrial and academic partners, coordinated by cosine and led by ESA. The entire production chain, from super-polished $300 \mathrm{~mm}$ wafers up to completed mirror module (see Figure 1) has been demonstrated and iterated, adjusting over time to different mission requirements in terms of focal length $(50 \mathrm{~m}, 20 \mathrm{~m}$, $12 \mathrm{~m}$ ) and sagittal radii $[7,8]$.
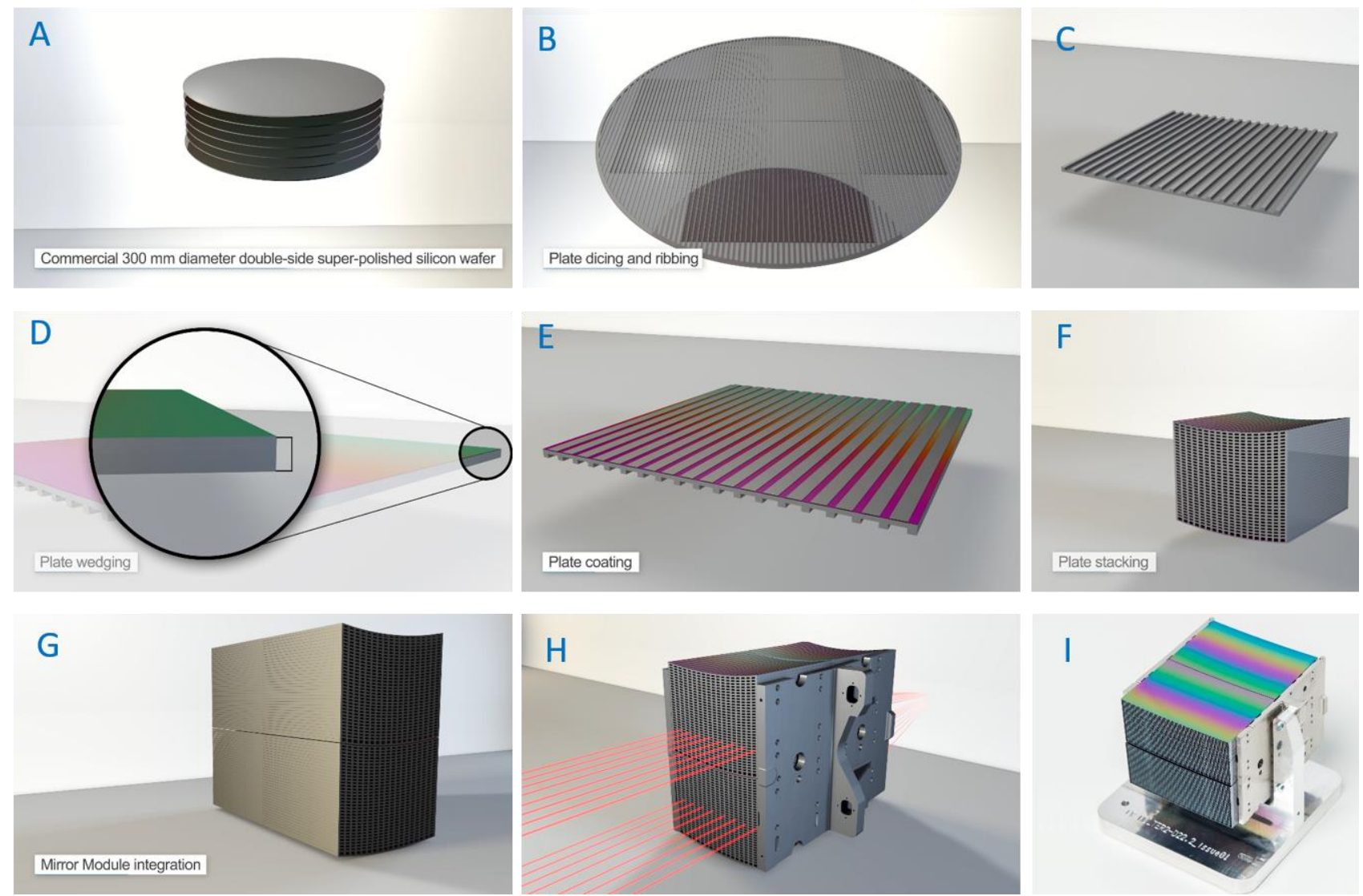

Figure 1: Production process of Silicon Pore Optics, starting with $300 \mathrm{~mm}$ silicon wafers (A). Those are ribbed and diced into plates (B, C), which are then wedged (D). The wedged plates are lithographically masked and coated $(E)$ and subsequently stacked using automated stacking robots $(F)$. Pairs of primary and secondary stacks are co-aligned using x-rays and glued into two invar brackets $(G, H)$, resulting in a completed mirror module (I).

The performance requirement for the angular resolution of Athena, expressed as half-energy width (HEW) of the pointspread function (PSF) at an energy of typically $1 \mathrm{keV}$, is usually the main criterion used to judge the status of the technology development. As the Athena optics has a radial span from $250 \mathrm{~mm}$ up to $1500 \mathrm{~mm}$, we develop and test SPO [9] at those two radii and in addition also at a middle radius of $750 \mathrm{~mm}$. Each radius demonstrates different aspects of the optics, from very long $(110 \mathrm{~mm})$ and narrow $(50 \mathrm{~mm})$ mirror plates at the innermost radius, to very short $(20 \mathrm{~mm})$, but wide $(102 \mathrm{~mm})$ plates of the outer mirror modules. X-ray testing of recent optics takes place at two facilities: One is the XPBF 2.0 (X-ray parallel beam facility) [10, 11], operated by PTB at the BESSY II synchrotron radiation facility. The other is the PANTER facility operated by the Max Planck Institut für extraterrestrische Physik (MPE) [12]. Double reflection measurements of a pair of SPO stacks (called an XOU) show that illuminating the central $70 \%$ of the width of the 10 plate XOU-0068 results at the nominal $12 \mathrm{~m}$ distance in a focus with a PSF of 7" HEW, measured at the PANTER facility [13], in good agreement with the BESSY x-ray data (see Figure 2). Part of that optic performs close to the 5" target. 
With this result achieved in January 2019 we increased the stack height to the nominal 34 plates, where in June 2019 we measured 10.2" HEW for the same central 70\% width (XOU-0078). 100\% of that optic had a PSF with a 13.9" HEW.
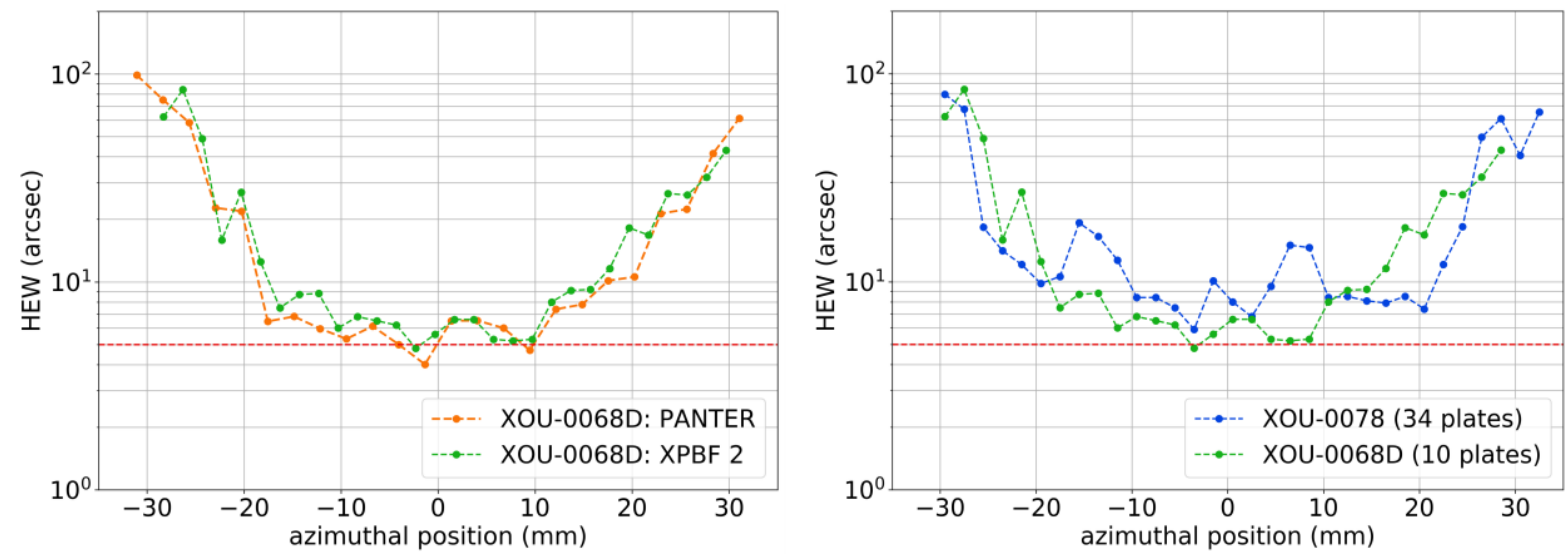

Figure 2: HEW as a function of scan position across the width of middle radii XOUs, being a pair of a primary and secondary stack measured in double reflection. Each data point is the measured HEW of 10 plate pairs (XOU-0068) and of 34 plate pairs (XOU-0078), along the entire length of the plates, across the entire height. The red dashed line indicates the 5" HEW goal of Athena. The left graph compares BESSY and PANTER measurements of $\mathrm{XOU}-0068$, showing very good agreement. The right graph compares 10 plate and 34 plate data of XOU-0068 and XOU-0078 measured at BESSY. The best central 70\% $(46 \mathrm{~mm})$ were measured with a HEW of 7.0" for XOU-0068 and of 10.2" for XOU-0078, at an energy of about $1.6 \mathrm{keV}$.

An extensive analysis, decomposing the different contributions to the PSF using newly developed metrology tools, showed that

A. the sides (first and last ribs) are degraded as a result of the tooling used in the stacking process.

B. the meridional curvature of XOU-0078 was too small, again a result of the tooling used in the stacking process.

C. systematic plate stains affect the edges of the mirror plates, a result of the wedging process and of cleaning carriers used in the wet-bench used before stacking.

D. the plates used in the stacks have a systematic wedge angle bias, which increases the total thickness variation (TTV), resulting in a stack-up error.

With these error terms identified as the largest contributors, we started in January 2019 to develop solutions for all of them. One should also note that many of these error terms found in middle radii stacks also apply to stacks made for the outer and inner radius, meaning that a solution developed for the middle radius can be transferred to the other radii. The next chapter discusses how we are improving the optics regarding points A, C \& D. The meridional curvature (point B) has already been addressed and is discussed elsewhere in these proceedings [14].

\section{CURRENT DEVELOPMENTS}

\section{Improving the sides of stacks}

To reduce the large slope deviations measured at the sides of stacks we are currently developing methods to create wider-than-nominal stacks, which are then reduced to their nominal width. This removes the problematic side regions by using so-called Sacrificial Lateral Extensions (SALEX) to the plates. Figure 3 shows a first proof-of-principle, where both the left and right side were removed after stacking, using fully industrial dicing systems.

Metrology, using the stacking robot fringe reflection technique (FRT) system, of the stack before and after removal shows no measurable degradation of the remaining area, and this is confirmed by $\mathrm{x}$-ray measurements. This paves the way to implement this method on a wider scale, creating stacks free of slope deviations along the entire width. Extrawide plates are being introduced, which shall become the basis of further developments. Until those have been fully 
implemented in the production process, one can rely on the $70 \%$ width $\mathrm{x}$-ray performance data to be representative for the future $100 \%$ performance of SPO mirror modules.
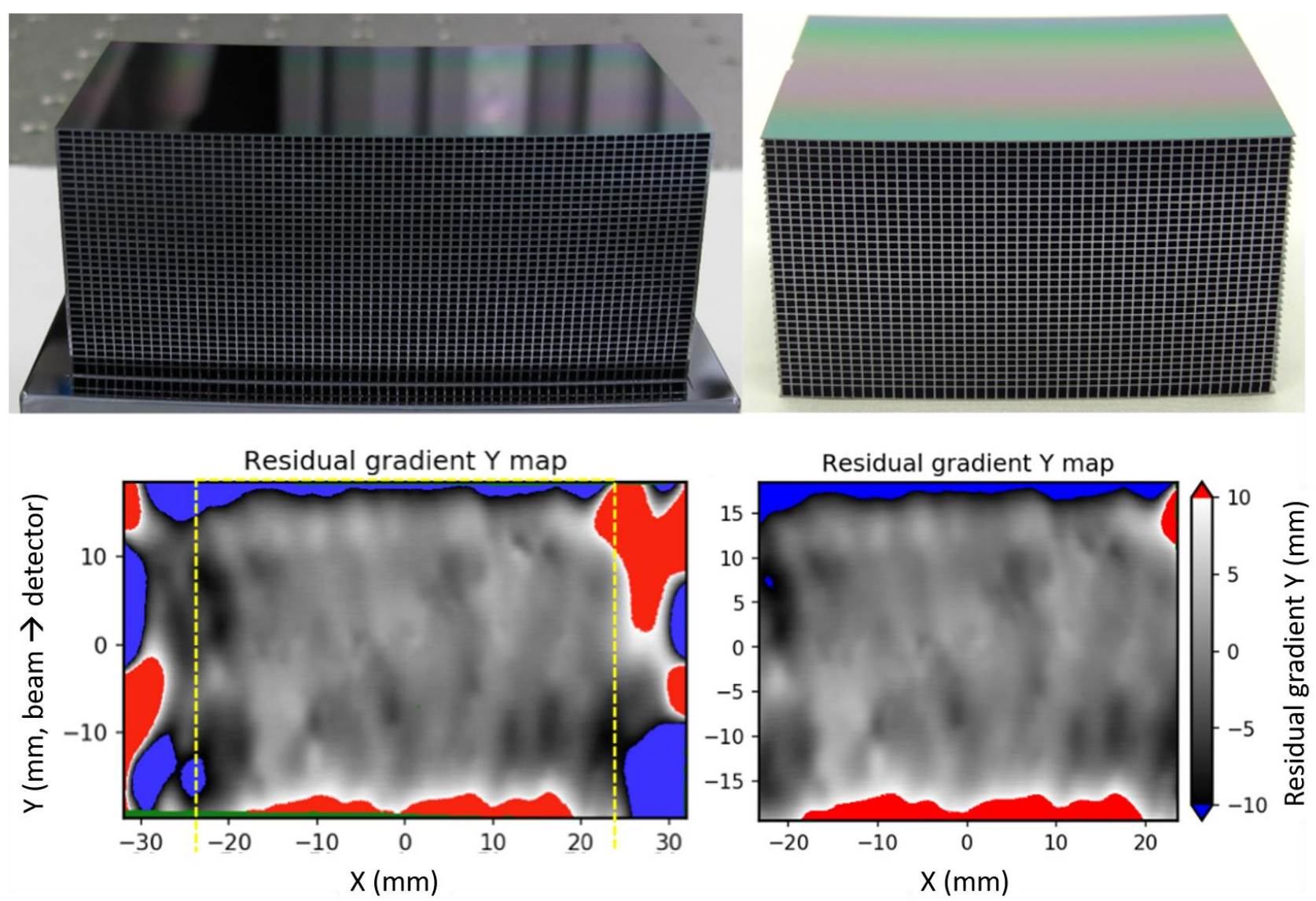

Figure 3: Top: photos of the same stack before (left) and after (right) the sides have been removed. Bottom: FRT metrology of the top of that 34 plate stack shows that post-stacking removal of the sides does not negatively affect the quality of the remaining parts of the optics.

\section{Improving the edges of the stacks}

Close to the edges the quality of the plates is degraded by some of the processing steps. One of these is the wedging process, which is required to make the plates in a stack confocal. This chemical process [15] leaves a few nanometer deep indents at certain fixed positions in a plate, which add up during the stacking process. Only a new developed metrology system, capable of measuring the TTV of plates to nanometer accuracy over $0.8 \mathrm{~mm}$ total thickness and with high spatial resolution, has been able to identify and quantify these defects, shown in Figure 4, as they would otherwise only become visible when combining the data of many different plates. The solution to this problem is similar to the one applied to the sides, namely to create extra-long plates and then shorten them to size. This method has now already been implemented for middle and outer radii plates, with a visible improvement as shown in Figure 4.

Another systematic issue, identified when we increased the production rate of stacks, are drying stains which also occur at fixed positions on the edges of the plates (see Figure 5). Those are a result of process carriers touching the plates. The effects are small on single plate level, but again stack up to significantly degrade the stack performance. We are in the process of developing new carriers and procuring a fully automated wet-bench, suitable for mass production of the Athena optics (right photograph in Figure 5). In combination with the aforementioned extra-wide SALEX-type plates we will be able to solve the problem of systematic drying stains, thereby further improving the performance of the optic. 

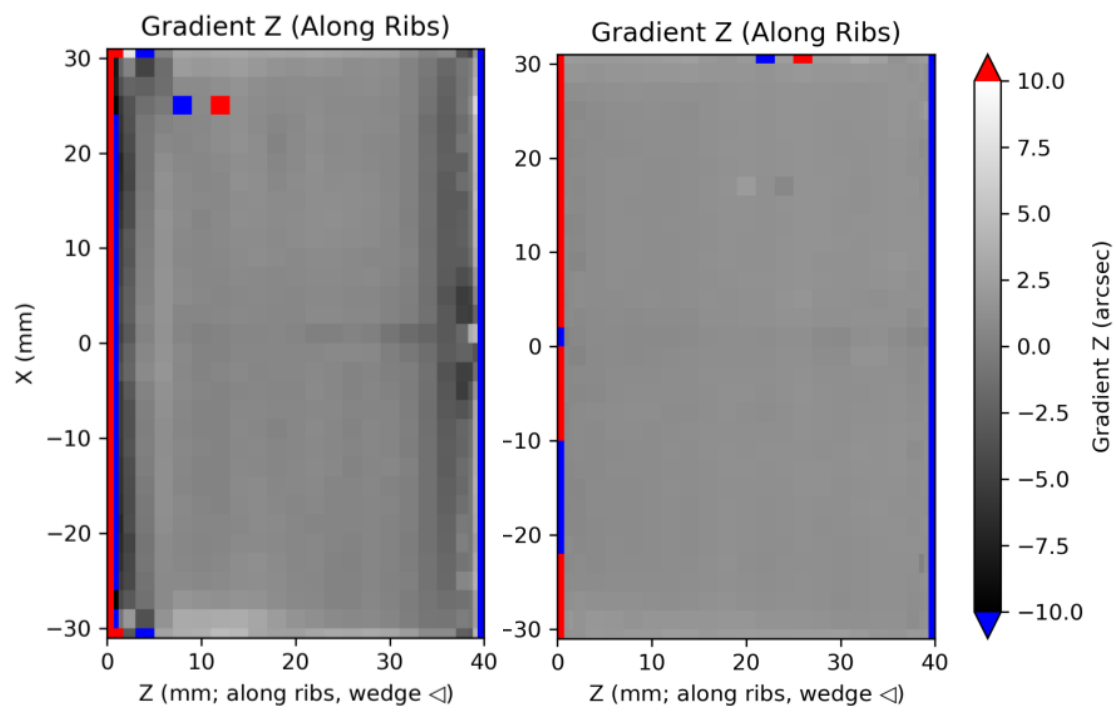

Figure 4: The sum of TTV measurements of 20 plates added on top of each other, showing the computed local gradient in $x$-ray ( $z$ ) direction. The left shows standard plates, revealing defects at the corners $(z=0 \mathrm{~mm}, x=-30 / 30$ $\mathrm{mm})$ and the centre of one edge $(\mathrm{z}=40 \mathrm{~mm}, \mathrm{x}=0 \mathrm{~mm})$. The right image shows added up data of 20 plates produced with the new process.
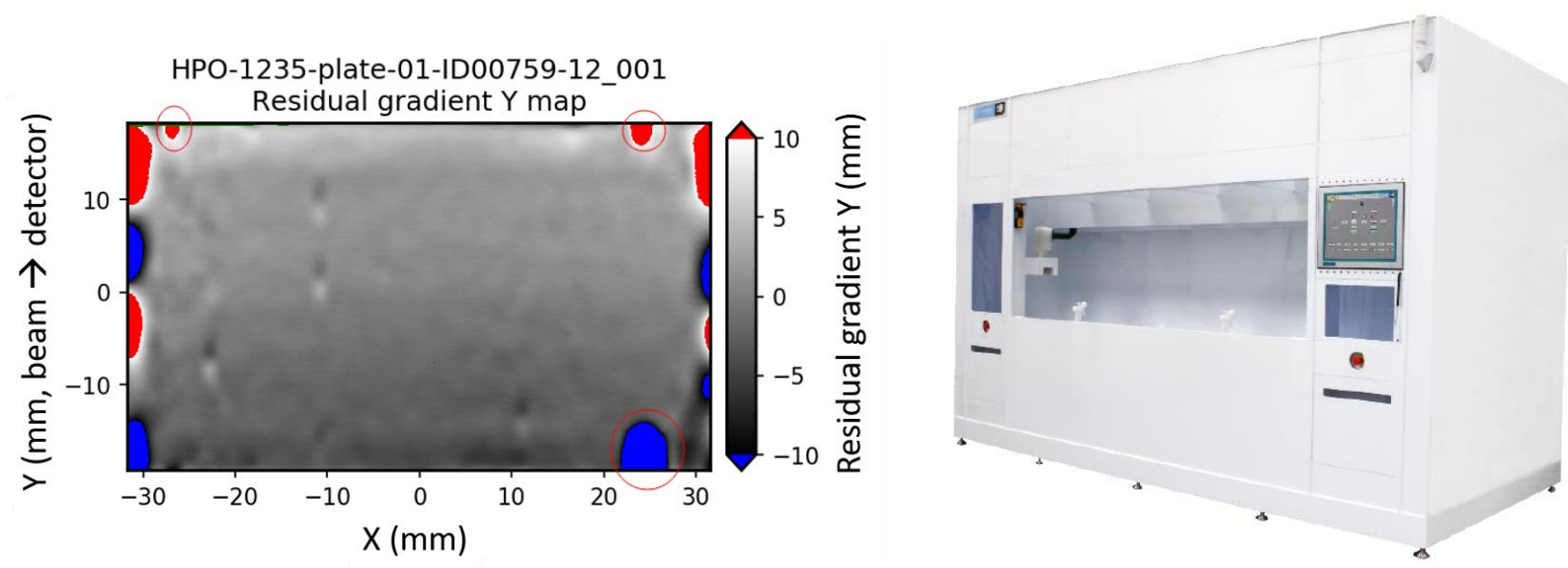

Figure 5: The left image shows a FRT metrology of a stacked plate, with three drying stains encircled in red. Those are caused by the cleaning carriers currently in use at the stacking facility. We currently are procuring a new, fully automated wet-bench (right photo), which will also include upgraded cleaning containers minimizing drying stains.

\section{Improving the wedge quality}

The aforementioned newly developed high-accuracy TTV measurement system also reveals a systematic wedge bias being present in the plates of one of the plate suppliers. The new metrology system can also measure the thickness of the wedge layer on the ribbed side, a measurement so far inaccessible to the plate supplier, due to limitations in their metrology. Whereas the wedge angle measurements of the reflective side of a plate are in good agreement and within the allowed specification (see Figure 6), the ribbed side has systematically a too large wedge angle, creating a bias when stacking the plates as the sum of both angles is too large. This systematic error can be reduced by adjusting the wedging process, bringing the combined total wedge angle per plate to the nominal value. The plate supplier has started to address this issue and we expect to soon receive plates that allow us to further reduce the stack up error. 


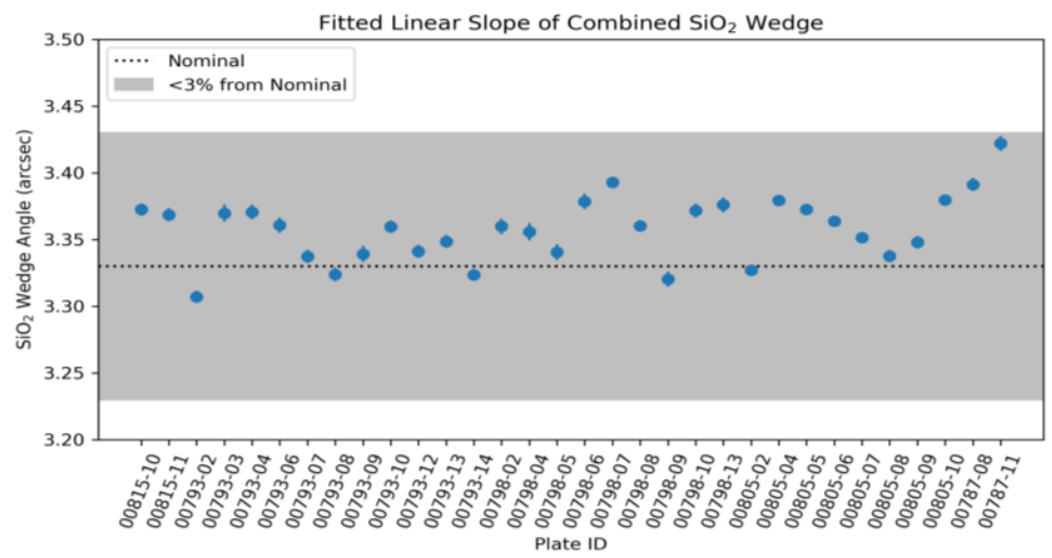

Figure 6: TTV measurements of 30 plates, using a newly developed metrology system. Those show the combined wedge angle of the plates. The wedge angle of the ribbed side is systematically too large, creating a bias in the combined wedge angle. This results in a systematic error component, when the plates are stacked up. The wedging process is now being adjusted to remove this bias and thus the associated HEW contribution of a few arc-seconds.

\section{STACKING PROCESS HARMONISATION}

All results presented in these proceedings so far have been obtained on middle radii stacks (for historic reasons the middle radius is the most advanced). Over the last years we also worked on the inner and outer radii, each being stacked on their own dedicated stacking robot. The robots were set up in the course of several years, with the outer stacking robot being the most recent addition. We started in 2019 the process of harmonizing the robots, such that all essential metrology and automation is the same. This allows us to apply the new stacking recipes, developed on one radius, to the other two radii.
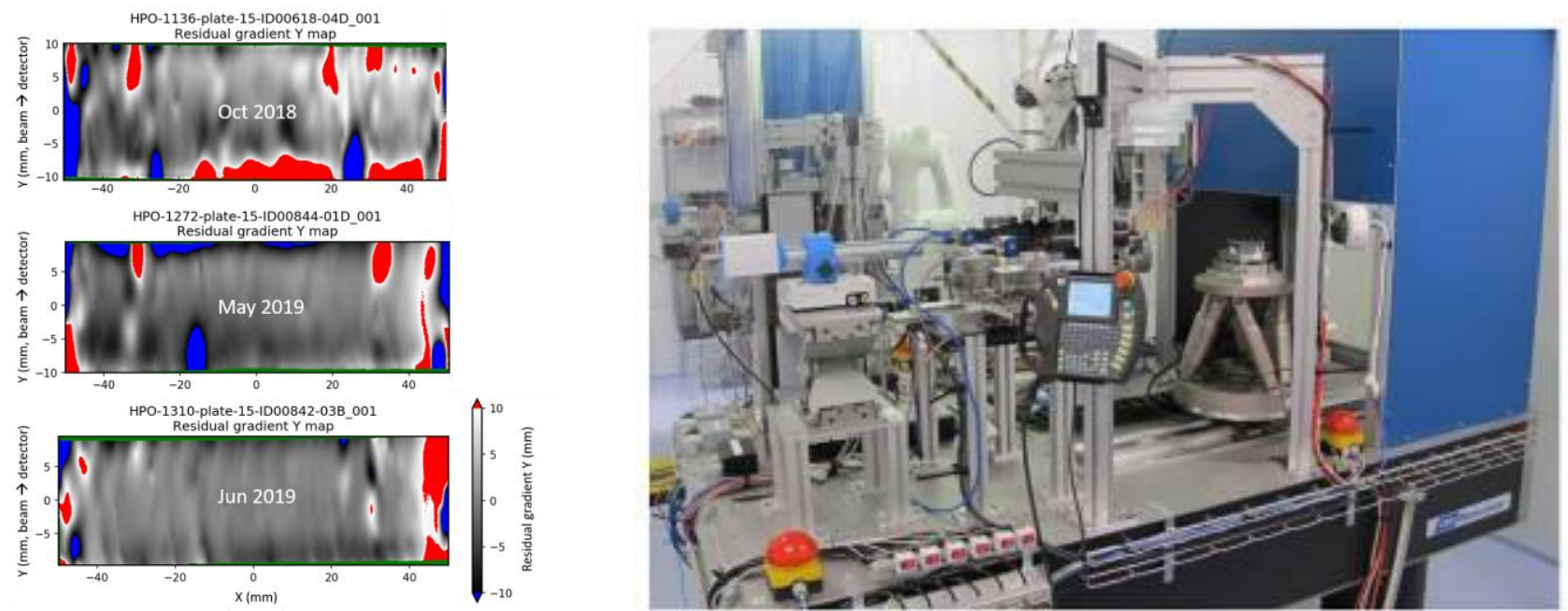

Figure 7: The left picture shows the evolution of the outer radius stack quality, comparing the FRT data of the $15^{\text {th }}$ plate of three stacks, as a function of time. The stacking recipe, developed on the middle radius robot, has been successfully migrated to the outer radius and significantly improved the quality of these stacks. The right picture shows a fourth stacking robot, developed and commissioned in 2019, which was installed in the cosine cleanroom. This robot will be dedicated for experimental work, whereas the other three robots are intended for continuous production on all three development radii.

One example is shown in the left of Figure 7, where we show the evolution of the quality of the outer radius stacks over the course of 9 months, achieved by implementing stacking recipes that were developed for the middle radius stacking 
robot. The improvement in quality is clearly visible and becomes comparable to the performance achieved on the middle radius.

For inner radii we are currently developing new dies that implement the improved processes of middle and outer radii. The stacking robot will be updated and we expect first improved results towards the first quarter of 2020 .

We also designed and built in 2019 a $4^{\text {th }}$ independent stacking robot, which is used to explore SPO developments that are currently outside of the main Athena development funded by ESA. Such developments, carried out by cosine for SRON and MPE, are for example the roughening of the backside of the plates [16] and the testing of baffling options to reduce straylight, and the increase of rib spacing to improve the field of view of the stacks.

In addition to the harmonization of the stacking robots, each development radius has also its own challenges in terms of plate geometry, wedging parameters and there are historical differences in plate membrane thickness and rib spacing. All of those will also be harmonized in the course of 2020, to prepare for the Mission Adoption Review (MAR) of Athena planned for the end of $2021[17,18]$.

\section{MASS PRODUCTION PREPARATIONS}

Parallel to the stacking process development we are ramping up the rate of production of the optics. Repeating a stacking recipe several times, with the goal to produce exactly the same stack, shows residual variations in the production parameters, which can subsequently be addressed. These variations can for example be small differences in the cleaning fluid concentrations, or differences in the wedge quality, as discussed above.

In order to produce more stacks we first had to increase the plate production rate. We introduced a second plate supplier and completed early 2019 the first step of the process by successfully receiving a batch of plates of all development radii for testing. The plate quality was found to be similar to the one of the first supplier and we will continue in 2019 to homogenise the plate quality received from the two suppliers. The plate production rate has increased to about 2000 plates per year, which meant that we also had to speed up the incoming inspection process at the stacking facility. This required automated measurement systems, such as for example the new TTV measurement system mentioned above, to be developed. It also requires the development of more cost effective and better plate packaging and transportation systems, which can be loaded efficiently with up to 80 plates in one go. The first prototype of such a transport container has been successfully tested and will be rolled out in 2020 .

In 2018 we commissioned a fully automated plate coating machine [19, 20], discussed elsewhere in these proceedings. It is already able to coat up to 100.000 plates per year. To prepare the plates for coating we require at the plate suppliers to include lithographic masking, using spray-coating of photo-resist as baseline process. This process is currently not yet automated and will need to be further improved by installing dedicated machines at the plate suppliers, planned for 2020. The lift-off process, taking place at the stacking facility, also needs to be automated and the newly designed wet-bench (see Figure 5) will include an automated lift-off bath, to become available in the second quarter of 2020. All of these measures taken together should bring the coated plate production rate up and further increase the plate production capacity at both suppliers, in line with the smooth ramp-up sought for the Athena flight model production.

The mirror module assembly speed is also being increased, with the goal of demonstrating one mirror module being produced on one synchrotron radiation beamline within $24 \mathrm{~h}$ [21]. This required the development of a fully motorized assembly jig that can manipulate 4 stacks in vacuum in 24 degrees of freedom. We will produce in the coming 9 months at least 6 mirror modules, which will not only be used to improve the assembly process, but will be also subject to environmental testing to complete the shock tests on all three radii [22].

\section{CONCLUSION}

Silicon Pore Optics has become a very mature x-ray optics technology, thanks to the continuous development efforts to prepare the industrial production of the largest $\mathrm{x}$-ray optics yet to be launched into space. With the selection of Athena as the next L2 mission in the ESA Cosmic Vision program the efforts have been intensified and we are now able to produce optics for the three development radii, representative for the final Athena configuration. Having demonstrated already all parts of the production chain, the technology development now focuses on improving the performance, and aims at ramping up the production rate in parallel. The central parts of the mirror stacks approach the 5" HEW angular resolution requirement and steps to increase the effective area to get within specification are part of a dedicated ESA technology development program to pass the Mission Adoption Review (MAR) milestone for Athena in 2021. 


\section{ACKNOWLEDGMENTS}

We would like to acknowledge all of the excellent and hard work of the many people and institutions that contribute to the development of Silicon Pore Optics. Without their efforts, dedication and talent we would not be able to build the lens required for the largest x-ray observatory to be flown so far. We also like to acknowledge the ESA, NWO (Athena, 184.034.002) and MPE funding that makes the development of SPO possible.

\section{REFERENCES}

[1] Beijersbergen, M. et al., "Silicon pore optics: novel lightweight high-resolution X-ray optics developed for XEUS", Proc. SPIE 5488, 868-874 (2004).

[2] Bavdaz, M. et al., "Progress at ESA on high-energy optics technologies", Proc. SPIE 5168, 136-147 (2004).

[3] K. Nandra, "Athena: Exploring the Hot and Energetic Universe", Proc. American Astronomical Society HEAD meeting (\#14), (2014).

[4] Ayre, M., et al, "ATHENA: system studies and optics Accommodation", Proc. of SPIE Vol. 10699, 106991E (2018)

[5] Willingale, R. et al, "Science requirements and optimization of the silicon pore optics design for the ATHENA mirror", Proc. of SPIE Vol. 9144, 91442E (2014)

[6] Wille, E., et al, "Silicon pore optics manufacturing plan and schedule for ATHENA", Proc. of SPIE Vol. 10699, 106993L (2018)

[7] Collon, M. J. et al, "Stacking of silicon pore optics for IXO", Proc. SPIE, 7437 (2009).

[8] Bavdaz, M. et al., "ESA led ATHENA/IXO optics development status", Proc. of SPIE 8147, 81470C1(2011).

[9] Vacanti, G, et al., "X-ray testing of silicon pore optics", in these proceedings of SPIE 11119-17 (2019).

[10] Krumrey, M., et al, "X-ray pencil beam facility for optics characterization", Proc. SPIE 7732, 773240 (2010).

[11] Krumrey, M., et al, "New X-ray parallel beam facility XPBF 2.0 for the characterization of silicon pore optics", Proc. SPIE 9905 (2016).

[12] Burwitz, V. et al., "Developing, testing, and calibrating the ATHENA optics at PANTER", Proc. SPIE 10399, 1039900 (2017).

[13] Bradshaw, M. J., et al, "Developments in testing x-ray optics at MPE's PANTER facility", in these proceedings of SPIE 11119-42 (2019).

[14] Keek, L., et al, "Stacking of mirrors for silicon pore optics", in these proceedings of SPIE 11119-16 (2019).

[15] Landgraf, B., et al, "Development and manufacturing of SPO x-ray mirrors", in these proceedings of SPIE 11119-13 (2019).

[16] Collon, M., et al, "Silicon Pore Optics Mirror Module Production and Testing", Proc. of SPIE Vol. 11180, 1118023 (2018).

[17] Bavdaz, M., et al, "Optics developments for ATHENA", in these proceedings of SPIE 11119-12 (2019).

[18] Ferreira, I. et al., "ATHENA: phase A study status and optics/instrument accomodation", in these proceedings of SPIE 11119-12 (2019).

[19] Massahi, S., et al, "Installation and commissioning of the silicon pore optics coatings facility for the Athena mission", in these proceedings of SPIE 11119-14 (2019).

[20] Svendsen, S., et al, "Performance and stability of $\mathrm{Ir} / \mathrm{SiC} \mathrm{x}$-ray mirror coatings for ATHENA", in these proceedings of SPIE 11119-15 (2019).

[21] Barrière, N. M., et al, "Assembly of confocal silicon pore optic mirror modules", in these proceedings of SPIE 11119-18 (2019).

[22] Girou, D., et al, "Environmental testing of silicon pore optics for Athena", in these proceedings of SPIE 1111919 (2019). 